ESAIM: M2AN 47 (2013) 1765-1781

DOI: $10.1051 / \mathrm{m} 2 \mathrm{an} / 2013087$
ESAIM: Mathematical Modelling and Numerical Analysis

www.esaim-m2an.org

\title{
CONSERVATION SCHEMES FOR CONVECTION-DIFFUSION EQUATIONS WITH ROBIN BOUNDARY CONDITIONS *,**
}

\author{
Stéphane Flotron ${ }^{1}$ and Jacques Rappaz ${ }^{1}$
}

\begin{abstract}
In this article, we present a numerical scheme based on a finite element method in order to solve a time-dependent convection-diffusion equation problem and satisfy some conservation properties. In particular, our scheme is able to conserve the total energy for a heat equation or the total mass of a solute in a fluid for a concentration equation, even if the approximation of the velocity field is not completely divergence-free. We establish a priori errror estimates for this scheme and we give some numerical examples which show the efficiency of the method.
\end{abstract}

Mathematics Subject Classification. 65M60,35K20, 80A20.

Received March 6, 2012. Revised April 23, 2013.

Published online October 11, 2013.

\section{INTRODUCTION}

A standard numerical method for the approximation of the solution of a time dependent convection-diffusion equation for a variable $\varphi$ transported with incompressible velocity $\boldsymbol{u}$, consists in multiplying the full equation by a space dependent test function $\psi$, in integrating it on the computational domain $\Omega$, and in discretizing it in space with a finite element method and in time with a finite difference scheme. The diffusion term is integrated by parts on $\Omega$ unlike the advected term $\boldsymbol{u} \cdot \boldsymbol{\nabla} \varphi$. The velocity field $\boldsymbol{u}$ is approximated by a velocity field $\boldsymbol{u}_{h}$ which is not completely divergence-free. For this reason, the standard numerical method does not preserve the energy or the mass when we model and approximate the evolution of the temperature or a concentration. In the convection dominated regime, a streamline upwind method SUPG is used in order to stabilize the numerical scheme, but it has no action on this advected term.

More precisely, when the flow is incompressible and confined in $\Omega$, i.e. when $\operatorname{div}(\boldsymbol{u})=0$ in $\Omega$ and $\boldsymbol{u} . \boldsymbol{n}=0$ on the boundary $\partial \Omega$, the integral of the variable $\varphi$ on the domain $\Omega$ remains constant in time when the source term is vanishing and when Neumann boundary conditions are applied on the boundary (conservation of the mass balance). When Robin boundary conditions are applied on the boundary $\partial \Omega$, as for example in a convectiondiffusion thermal problem, an energy mass balance can be established by taking into account the energy crossing through $\partial \Omega$.

Keywords and phrases. Finite Elements, numerical conservation schemes, Robin boundary condition, convection-diffusion equations.

* Supported by Rio-Tinto Alcan Company.

** We thank A. Caboussat for the correction of the English.

1 Ecole Polytechnique Fédérale de Lausanne, 1015 Lausanne, Switzerland.

stephane.flotron@epfl.ch; jacques.rappaz@epfl.ch 
From a practical viewpoint, the velocity $\boldsymbol{u}$ is often computed with a Navier-Stokes solver which leads to an approximation $\boldsymbol{u}_{h}$ which is not exactly (elementwise) divergence free. As an unwelcome numerical effect, the mass balance or the energy balance are not conserved when the time increases. These losses can be important when the equation is integrated on a long time. In this article, we present an original modification of the standard numerical scheme in order to eliminate this drawbacks which appears when Neumann or Robin boundary conditions for $\varphi$ are imposed on $\partial \Omega$. We show that this novel scheme is $L^{2}$-stable and allows to obtain a constant stationary solution when the source term is vanishing. We also establish some error estimates produced by this new scheme.

Let us mention that the discretization of the convection term has been widely studied, due to the fact that it has not all desired properties. For example, in [11], Temam studied a discretization of convection which is $L^{2}$-stable, because the standard discretization doesn't have this property. Another example is the combination of a finite element method and a finite volume method in order to conserve the numerical fluxes, as done in [1]. However, this approach has the major drawback that two grids coexist during the computation: one for the finite element method and one for the finite volume method. The method that we propose here doesn't suffer from this drawback, can easily be implemented, and ensures the conservation of the numerical fluxes on the boundary of the domain.

\section{Statement of the PROBlem}

Let us consider a cavity $\Omega \subset \mathbb{R}^{3}$ bounded and with a boundary $\partial \Omega$ Lipschitzian. An incompressible fluid flows in this cavity, with velocity $\boldsymbol{u}$ depending on $t \in(0, \infty)$ and $\boldsymbol{x} \in \Omega$, while a passive scalar or a temperature field $\varphi$ is convected and diffused. If $\boldsymbol{n}$ is the external unit normal to the domain $\Omega$, we assume that

$$
\operatorname{div}(\boldsymbol{u})=0 \text { in } \Omega \text { and } \boldsymbol{u} \cdot \boldsymbol{n}=0 \text { on } \partial \Omega,
$$

where $\boldsymbol{u} . \boldsymbol{n}$ is the Euclidean scalar product of $\boldsymbol{u}$ with $\boldsymbol{n}$.

The convection-diffusion equation for $\varphi$ is given by:

$$
\frac{\partial \varphi}{\partial t}-\epsilon \Delta \varphi+\boldsymbol{u} \cdot \nabla \varphi=f \text { in }(0,+\infty) \times \Omega,
$$

with Robin boundary condition:

$$
\epsilon \frac{\partial \varphi}{\partial n}=\alpha\left(\varphi_{r}-\varphi\right) \text { on } \partial \Omega
$$

and initial condition

$$
\varphi=\varphi_{0} \text { at time } t=0,
$$

where $\varphi_{r}$ is a given constant number and $\alpha$ is a non negative parameter. In equation (2.2), $f$ is a source term that depends on $t \in(0,+\infty)$ and $\boldsymbol{x} \in \Omega$, and $\epsilon>0$ is the diffusion coefficient.

Let us observe that it is not restrictive to assume that $\varphi_{r}=0$ since it suffices to change the unknown $\varphi$ onto $\left(\varphi-\varphi_{r}\right)$. Thus, in this sequel we assume that $\varphi_{r}=0$.

From a mathematical point of view, we assume that $T>0$ is the final time and that $f \in L^{2}((0, T) \times \Omega)$ and $\varphi_{0} \in L^{2}(\Omega)$. Using the standard notations for Sobolev spaces $H^{1}(\Omega), H^{2}(\Omega), H^{1}\left((0, T) ; L^{2}(\Omega)\right), C^{1}([0, T]$; $L^{2}(\Omega)$ ), (see [6,7]), we assume that $\boldsymbol{u} \in H^{2}(\Omega)^{3}$ is given and not depending on $t$ (in fact it is not difficult to adapt the following discussion to the case where $\boldsymbol{u}$ is depending on $t$ ).

A classical weak formulation of $(2.2)-(2.3)$ with $\varphi_{r}=0$ (see $[6,10]$ ) consists in looking for $\varphi \in$ $L^{2}\left((0, T) ; H^{1}(\Omega)\right) \cap C^{0}\left([0, T] ; L^{2}(\Omega)\right)$ satisfying for every $\psi \in H^{1}(\Omega)$ :

$$
\int_{\Omega} \frac{\partial \varphi}{\partial t} \psi \mathrm{d} x+\epsilon \int_{\Omega} \boldsymbol{\nabla} \varphi \cdot \nabla \psi \mathrm{d} x+\alpha \int_{\partial \Omega} \varphi \psi \mathrm{d} s+\int_{\Omega}(\boldsymbol{u} \cdot \boldsymbol{\nabla} \varphi) \psi \mathrm{d} x=\int_{\Omega} f \psi \mathrm{d} x
$$


Since we have assumed that $\operatorname{div}(\boldsymbol{u})=0$, then $\boldsymbol{u} \cdot \boldsymbol{\nabla} \varphi=\operatorname{div}(\boldsymbol{u} \varphi)$ and $(\boldsymbol{u} \cdot \boldsymbol{\nabla} \varphi) \varphi=\frac{1}{2} \operatorname{div}\left(\boldsymbol{u} \varphi^{2}\right)$. Moreover with $\boldsymbol{u} . \boldsymbol{n}=0$ on $\partial \Omega$, we obtain by using the divergence theorem:

Property 1. If $\psi=1$ in (2.5), we have:

$$
\frac{\mathrm{d}}{\mathrm{d} t} \int_{\Omega} \varphi \mathrm{d} x+\alpha \int_{\partial \Omega} \varphi \mathrm{d} s=\int_{\Omega} f \mathrm{~d} x .
$$

This property is important since it describes the conservation of the thermal energy if $\varphi$ is a temperature variable or the conservation of the mass of material if $\varphi$ is a density variable. For example if the source term is vanishing and if the physical system is isolated $(f=0$ and $\alpha=0)$, the integral of $\varphi$ on $\Omega$ remains constant in time (conservation of total energy or conservation of total mass in $\Omega$, i.e. $\int_{\Omega} \varphi \mathrm{d} x=\int_{\Omega} \varphi_{0} \mathrm{~d} x$ for every $t>0$ ).

Let us now denote by $\|v\|$ the $L^{2}(\Omega)$ norm of $v \in L^{2}(\Omega)$ and $\|v\|_{1}=\operatorname{def}_{\Omega}\left(\epsilon \int_{\Omega}|\nabla v|^{2} \mathrm{~d} x+\alpha \int_{\partial \Omega}|v|^{2} \mathrm{~d} s\right)^{\frac{1}{2}}$ for $v \in H^{1}(\Omega)$. Taking $\psi=\varphi$ in (2.5), we have

$$
\frac{1}{2} \frac{\mathrm{d}}{\mathrm{d} t}\|\varphi\|^{2}+\|\varphi\|_{1}^{2}=\int_{\Omega} f \varphi \mathrm{d} x
$$

If $\lambda_{1}=\inf _{v \in H^{1}(\Omega)} \frac{\|v\|_{1}^{2}}{\|v\|^{2}}$, by using the Cauchy-Schwarz inequality, we obtain

\section{Property 2.}

$$
\frac{\mathrm{d}}{\mathrm{d} t}\|\varphi\|+\lambda_{1}\|\varphi\| \leq\|f\| .
$$

In particular, if $\alpha>0$, then $\|\cdot\|_{1}$ is a norm equivalent to the standard $H^{1}$ norm and $\lambda_{1}$ is strictly positive. Moreover, when $f=0$, the variable $\varphi$ is exponentially decreasing and $\|\varphi\|=\mathrm{e}^{-\lambda_{1} t}\left\|\varphi_{0}\right\|$. In the case $\alpha=0$, we obtain the same behavior for $\varphi-\bar{\varphi}$ where $\bar{\varphi}$ is the mean value of $\varphi$ in $\Omega$. Finally we have

\section{Property 3.}

$$
\text { if } \alpha=0 \text { and } f=0 \text {, then } \varphi=\text { constant is a stationary solution of }(2.5)
$$

Let us remark that in these three properties, the velocity $\boldsymbol{u}$ has no influence since it is divergence-free.

In the next section, given an approximation $\boldsymbol{u}_{h}$ of $\boldsymbol{u}$, we would like to define a semi-discretization in space of (2.5) (by taking $\varphi_{h} \in V_{h} \subset H^{1}(\Omega)$ ) that allows to compute an approximation $\varphi_{h}$ of $\varphi$ that satisfies the above properties, i.e.

Property 1h. conservation of the integral of $\varphi_{h}$ :

$$
\frac{\mathrm{d}}{\mathrm{d} t} \int_{\Omega} \varphi_{h} \mathrm{~d} x+\alpha \int_{\partial \Omega} \varphi_{h} \mathrm{~d} s=\int_{\Omega} f \mathrm{~d} x .
$$

Property 2h. $L^{2}$-stability of the scheme:

$$
\frac{\mathrm{d}}{\mathrm{d} t}\left\|\varphi_{h}\right\|+\lambda_{1 h}\left\|\varphi_{h}\right\| \leq\|f\|
$$

where $\lambda_{1 h}=\inf _{v_{h} \in V_{h}} \frac{\left\|v_{h}\right\|_{1}^{2}}{\left\|v_{h}\right\|^{2}}$. Let us mention that in a standard finite element method, we have $\lambda_{1 h} \geq \lambda_{1}$ (see [2] p. 699), which implies that

$$
\frac{\mathrm{d}}{\mathrm{d} t}\left\|\varphi_{h}\right\|+\lambda_{1}\left\|\varphi_{h}\right\| \leq\|f\|
$$

\section{Property 3h. stationary constant solution:}

$$
\text { if } \alpha=0 \text { and } f=0 \text {, then } \varphi_{h}=\text { constant is a stationary solution. }
$$




\section{Semi-Discretization In SPACE}

In order to consider a semi-discretization in space of Equation (2.5), we assume for the sake of simplicity, that $\Omega$ is a polyhedral domain. If $\Gamma_{h}$ is a conforming mesh of $\Omega$ composed by tetrahedra $K \in \Gamma_{h}$ with diameter $h_{K}$ smaller than $h$, we define the standard finite element space $V_{h}$ of piecewise polynomial functions $\mathbb{P}_{1}(K)$ of degree 1 on $K$ by

$$
V_{h}=\left\{g: \Omega \rightarrow \mathbb{R}: g \text { continuous and }\left.g\right|_{K} \in \mathbb{P}_{1}(K), \forall K \in \Gamma_{h}\right\} .
$$

When $h_{K}$ is small with respect to $\epsilon /\left\|\boldsymbol{u}_{h}\right\|_{L_{2}(K)}$ for every $K \in \Gamma_{h}$, a standard finite element approximation scheme in space for computing an approximation $\varphi_{h}$ of $\varphi$ is to look for a function $\varphi_{h} \in H^{1}\left((0, T) ; V_{h}\right)$ satisfying:

$$
\int_{\Omega} \frac{\partial \varphi_{h}}{\partial t} \psi_{h} \mathrm{~d} x+\epsilon \int_{\Omega} \nabla \varphi_{h} \cdot \nabla \psi_{h} \mathrm{~d} x+\alpha \int_{\partial \Omega} \varphi_{h} \psi_{h} \mathrm{~d} s+\int_{\Omega} L\left(\boldsymbol{u}_{h}, \varphi_{h}, \psi_{h}\right) \mathrm{d} x=\int_{\Omega} f \psi_{h} \mathrm{~d} x, \quad \forall \psi_{h} \in V_{h},
$$

where $\boldsymbol{u}_{h} \in V_{h}^{3}$ is an approximation of $\boldsymbol{u}$ obtained for instance with a finite element Navier-Stokes code, and $\int_{\Omega} L\left(\boldsymbol{u}_{h}, \varphi_{h}, \psi_{h}\right) \mathrm{d} x$ is a discretization of $\int_{\Omega}(\boldsymbol{u} \cdot \boldsymbol{\nabla} \varphi) \psi \mathrm{d} x$. The most popular approximation of $\int_{\Omega}(\boldsymbol{u} \cdot \boldsymbol{\nabla} \varphi) \psi \mathrm{d} x$ is obtained by setting $L\left(\boldsymbol{u}_{h}, \varphi_{h}, \psi_{h}\right)=\left(\boldsymbol{u}_{h} \cdot \nabla \varphi_{h}\right) \psi_{h}$.

In (3.2) we assume that the initial condition $\varphi_{0}$ is given in $V_{h}$ and if it is not the case, we take a projection $\varphi_{h}^{0} \in V_{h}$ of $\varphi_{0}$ as initial condition $\varphi_{h}(0)$.

Of course if $h_{K}$ is greater than $\epsilon /\left\|\boldsymbol{u}_{h}\right\|_{L_{2}(K)}$ for some $K \in \Gamma_{h}$ (convection dominated regime in a neighborhood of $K$ ), an artificial term, SUPG-like is added to (3.2) (see [3]) which is

$$
\omega \sum_{K \in \Gamma_{h}} \frac{\tau_{K} h_{K}}{2\left\|\boldsymbol{u}_{h}\right\|_{L_{2}(K)}} \int_{K}\left(\boldsymbol{u}_{h} \cdot \boldsymbol{\nabla} \varphi_{h}\right)\left(\boldsymbol{u}_{h} \cdot \nabla \psi_{h}\right) \mathrm{d} x .
$$

This term allows to eliminate some spurious numerical oscillations. In (3.3), $\omega$ is an appropriate constant and $\tau_{K}=\max \left(0,1-2 \epsilon / h_{K}\left\|\boldsymbol{u}_{h}\right\|_{L_{2}(K)}\right)$. Another possibility to eliminate spurious numerical oscillation is to add to (3.2) an edge stabilization (see [4]). In the following we neglect the addition of these artificial terms which have no influence on our conclusions.

Let us assume that $\boldsymbol{u}_{h}$ is an approximation of $\boldsymbol{u}$ with the following properties: there exists a constant $C$ such that

$$
\left\|\boldsymbol{u}-\boldsymbol{u}_{h}\right\|+h\left\|\nabla\left(\boldsymbol{u}-\boldsymbol{u}_{h}\right)\right\| \leq C h^{2} .
$$

and

$$
\boldsymbol{u}_{h} \cdot \boldsymbol{n}=0 \text { on } \partial \Omega .
$$

Even if $\operatorname{div}\left(\boldsymbol{u}_{h}\right)$ is not vanishing but only of order $h$ in the $L^{2}$-norm, we would like the trilinear functional $L:(\boldsymbol{u}, \varphi, \psi) \in H^{1}(\Omega)^{3} \times H^{1}(\Omega) \times H^{1}(\Omega) \rightarrow L(\boldsymbol{u}, \varphi, \psi) \in \mathbb{R}$ to satisfy the following properties, for consistency reasons and in order to satisfy $(2.10),(2.12),(2.13)$ :
1) $\int_{\Omega} L(\boldsymbol{u}, \varphi, \psi) \mathrm{d} x=\int_{\Omega}(\boldsymbol{u} . \nabla \varphi) \psi \mathrm{d} x, \forall \varphi, \psi \in H^{1}(\Omega) ;$
2) $\int_{\Omega} L\left(\boldsymbol{u}_{h}, \psi_{h}, 1\right) \mathrm{d} x=0 \quad \forall \psi_{h} \in V_{h}$;
3) $\int_{\Omega} L\left(\boldsymbol{u}_{h}, \psi_{h}, \psi_{h}\right) \mathrm{d} x=0 \quad \forall \psi_{h} \in V_{h}$;
4) $\int_{\Omega} L\left(\boldsymbol{u}_{h}, 1, \psi_{h}\right) \mathrm{d} x=0 \quad \forall \psi_{h} \in V_{h}$. 
TABLE 1. Conservation properties for different discretizations of the convective term $L$ when $\operatorname{div} \boldsymbol{u}_{h} \neq 0$.

\begin{tabular}{|l|c|c|c|}
\hline$L\left(\boldsymbol{u}_{h}, \varphi_{h}, \psi_{h}\right)$ & Property $1 h$ & Property $2 h$ & Property $3 h$ \\
\hline L1 & no & no & yes \\
L2 & yes & no & no \\
L3 & yes & no & no \\
L4 & no & yes & no \\
L5 & yes & yes & yes \\
\hline
\end{tabular}

In order to satisfy the consistency relation 1), the standard versions of $L$ for the discretization of $\int_{\Omega}(\boldsymbol{u} \cdot \boldsymbol{\nabla} \varphi) \psi \mathrm{d} x$ by $\int_{\Omega} L\left(\boldsymbol{u}_{h}, \varphi_{h}, \psi_{h}\right) \mathrm{d} x$ are the following:

L1) $L(\boldsymbol{u}, \varphi, \psi)=(\boldsymbol{u} . \nabla \varphi) \psi$,

L2) $L(\boldsymbol{u}, \varphi, \psi)=-(\boldsymbol{u} \cdot \nabla \psi) \varphi$,

L3) $L(\boldsymbol{u}, \varphi, \psi)=\operatorname{div}(\varphi \boldsymbol{u}) \psi$,

L4) $L(\boldsymbol{u}, \varphi, \psi)=\frac{1}{2}((\boldsymbol{u} \cdot \nabla \varphi) \psi-(\boldsymbol{u} \cdot \boldsymbol{\nabla} \psi) \varphi)$.

Unfortunately, none of these choices satisfies the relations 2),3),4) at the same time when $\operatorname{div} \boldsymbol{u}_{h}$ is not vanishing. Hence properties $1 \mathrm{~h}$ ) to $3 \mathrm{~h}$ ) cannot be satisfied simultaneously. A summary of the conservation properties is shown in Table 1. In this article, we advocate the following discretization of $L$ :

$$
\text { L5) } L(\boldsymbol{u}, \varphi, \psi)=\frac{1}{2}[(\boldsymbol{u} \cdot \boldsymbol{\nabla} \varphi)(\psi-\bar{\psi})-(\boldsymbol{u} \cdot \boldsymbol{\nabla} \psi)(\varphi-\bar{\varphi})]
$$

where the notation $\bar{\omega}=\frac{1}{\Omega \mid} \int_{\Omega} \omega \mathrm{d} x$ denotes the mean value of a function $\omega$ on $\Omega$. It is easy to verify that the above relations 1), 2), 3), and 4) are simultaneously satisfied with this choice and consequently the properties 1h), $2 \mathrm{~h}$ ) and $3 \mathrm{~h}$ ) are simultaneously satisfied with choice (L5), as shown in Table 1.

Replacing $L\left(\boldsymbol{u}_{h}, \varphi_{h}, \psi_{h}\right)$ by (L5) in Scheme (3.2), we advocate the following space approximation of (2.5): we are looking for $\varphi_{h} \in H^{1}\left((0, T) ; V_{h}\right)$ satisfying:

$$
\begin{gathered}
\int_{\Omega} \frac{\partial \varphi_{h}}{\partial t} \psi_{h} \mathrm{~d} x+\epsilon \int_{\Omega} \boldsymbol{\nabla} \varphi_{h} \cdot \boldsymbol{\nabla} \psi_{h} \mathrm{~d} x+\alpha \int_{\partial \Omega} \varphi_{h} \psi_{h} d s+\frac{1}{2} \int_{\Omega}\left(\boldsymbol{u}_{h} \cdot \boldsymbol{\nabla} \varphi_{h}\right)\left(\psi_{h}-\bar{\psi}_{h}\right) \mathrm{d} x \\
-\frac{1}{2} \int_{\Omega}\left(\boldsymbol{u}_{h} \cdot \boldsymbol{\nabla} \psi_{h}\right)\left(\varphi_{h}-\bar{\varphi}_{h}\right) \mathrm{d} x=\int_{\Omega} f \psi_{h} \mathrm{~d} x, \quad \forall \psi_{h} \in V_{h} .
\end{gathered}
$$

Remark 3.1. As said before, if we want to eliminate some spurious numerical oscillations in dominated convection problem, we can add a SUPG term of the form (3.3) in numerical scheme (3.6). We can easily show that this term has no influence on the conservation of the three desired properties. In particular, the addition of (3.3) into (3.6) increase the $L^{2}$-stability of the scheme and (2.12) still holds.

From a practical point of view, it is not convenient to work with Scheme (3.6). Indeed, the support of $\psi_{h}-\bar{\psi}_{h}$ is $\Omega$ and hence the matrix obtained by Scheme (3.6) is not sparse anymore and becomes full. To avoid this, we use a partition of the function space. More precisely, if $W$ is a space of integrable functions defined on $\Omega$, we denote by $\widetilde{W}=\left\{g \in W: \int_{\Omega} g \mathrm{~d} x=0\right\}$, and we use the partition $W=\widetilde{W} \oplus \mathbb{R}$. Hence, if $\omega \in W$, we set $\bar{\omega}=\frac{1}{|\Omega|} \int_{\Omega} \omega \mathrm{d} x$ and $\omega=\widetilde{\omega}+\bar{\omega}$ with $\bar{\omega} \in \mathbb{R}$ and $\widetilde{\omega}=\omega-\bar{\omega} \in \widetilde{W}$. Let us consider $\widetilde{\varphi}_{h} \in H^{1}\left((0, T) ; \widetilde{V}_{h}\right)$ and $\bar{\varphi}_{h} \in$ $H^{1}((0, T) ; \mathbb{R})$ solution of both equations:

$$
\begin{aligned}
\int_{\Omega} & \frac{\partial \widetilde{\varphi}_{h}}{\partial t} \psi_{h} \mathrm{~d} x+\epsilon \int_{\Omega} \boldsymbol{\nabla} \widetilde{\varphi}_{h} \cdot \nabla \psi_{h} \mathrm{~d} x+\alpha \int_{\partial \Omega}\left(\bar{\varphi}_{h}+\widetilde{\varphi}_{h}\right) \psi_{h} \mathrm{~d} s+\frac{1}{2} \int_{\Omega}\left(\boldsymbol{u}_{h} \cdot \boldsymbol{\nabla} \widetilde{\varphi}_{h}\right) \psi_{h} \mathrm{~d} x \\
& -\frac{1}{2} \int_{\Omega}\left(\boldsymbol{u}_{h} \cdot \boldsymbol{\nabla} \psi_{h}\right) \widetilde{\varphi}_{h} \mathrm{~d} x=\int_{\Omega} f \psi_{h} \mathrm{~d} x, \quad \forall \psi_{h} \in \widetilde{V}_{h},
\end{aligned}
$$


and

$$
\frac{\mathrm{d}}{\mathrm{d} t} \int_{\Omega} \bar{\varphi}_{h} \mathrm{~d} x+\alpha \int_{\partial \Omega}\left(\bar{\varphi}_{h}+\widetilde{\varphi}_{h}\right) \mathrm{d} s=\int_{\Omega} f \mathrm{~d} x
$$

with initial condition $\widetilde{\varphi}_{h}(0)=\varphi_{h}^{0}-\bar{\varphi}_{h}^{0}$, where $\varphi_{h}^{0}$ is an approximation of $\varphi_{0}$ and $\bar{\varphi}_{h}^{0}=\bar{\varphi}_{h}(0)=\frac{1}{|\Omega|} \int_{\Omega} \varphi_{h}^{0} \mathrm{~d} x$. Taking consecutively $\psi_{h} \in \widetilde{V}_{h}, \psi_{h} \equiv 1$ in (3.6) and using $\varphi_{h}=\widetilde{\varphi}_{h}+\bar{\varphi}_{h}$, we easily verify that Problem (3.6) and Problem (3.7)-(3.8) are equivalent.

In (3.7) the mean value of the test function $\psi_{h}$ is equal to zero, which is not standard in the finite element method. Thus this constrain is taken into account by a Lagrange multiplier $\lambda$. On the other hand we add an equation in order to impose $\int_{\Omega} \widetilde{\varphi}_{h} \mathrm{~d} x=0$. Consequently we are looking for $\widetilde{\varphi}_{h} \in H^{1}\left((0, T) ; V_{h}\right), \bar{\varphi}_{h} \in$ $H^{1}((0, T) ; \mathbb{R})$ and $\lambda \in H^{1}((0, T) ; \mathbb{R})$ satisfying:

$$
\begin{gathered}
\int_{\Omega} \frac{\partial \widetilde{\varphi}_{h}}{\partial t} \psi_{h} \mathrm{~d} x+\epsilon \int_{\Omega} \boldsymbol{\nabla} \widetilde{\varphi}_{h} \cdot \nabla \psi_{h} \mathrm{~d} x+\alpha \int_{\partial \Omega}\left(\bar{\varphi}_{h}+\widetilde{\varphi}_{h}\right) \psi_{h} \mathrm{~d} s+\frac{1}{2} \int_{\Omega}\left(\boldsymbol{u}_{h} \cdot \boldsymbol{\nabla} \widetilde{\varphi}_{h}\right) \psi_{h} \mathrm{~d} x \\
-\frac{1}{2} \int_{\Omega}\left(\boldsymbol{u}_{h} \cdot \boldsymbol{\nabla} \psi_{h}\right) \widetilde{\varphi}_{h} \mathrm{~d} x+\lambda \int_{\Omega} \psi_{h} \mathrm{~d} x=\int_{\Omega} f \psi_{h} \mathrm{~d} x, \quad \forall \psi_{h} \in V_{h}, \\
\frac{\mathrm{d}}{\mathrm{d} t} \int_{\Omega} \bar{\varphi}_{h} \mathrm{~d} x+\alpha \int_{\partial \Omega}\left(\bar{\varphi}_{h}+\widetilde{\varphi}_{h}\right) \mathrm{d} s=\int_{\Omega} f \mathrm{~d} x, \\
\int_{\Omega} \widetilde{\varphi}_{h} \mathrm{~d} x=0 .
\end{gathered}
$$

If the dimension of $V_{h}$ is $N$, then (3.9), (3.10) and (3.11) is a system of ordinary differential equation in time with $(N+2)$ equations, in which the unknowns $\widetilde{\varphi}_{h}, \bar{\varphi}_{h}$ and $\lambda$ are coupled. In the case $\alpha=0$ (Neumann boundary conditions) the unknown $\bar{\varphi}_{h}$ is not coupled to the other variables $\widetilde{\varphi}_{h}$ and $\lambda$. In conclusion, Problem (3.6) is equivalent to (3.9), (3.10), (3.11), but on a practical point of view, this last formulation is easier to solve than the previous one.

\section{ERror ESTIMATES}

Now we establish error bounds between $\varphi$ and $\varphi_{h}$ in various norms, when $\varphi_{h}$ is solution of (3.6). To do this, we follow [12] and assume the realistic hypothesis (3.4) on the velocity field $\boldsymbol{u}$ and its approximation $\boldsymbol{u}_{h}$.

Let us remark that estimate (3.4) holds in a lot of standard finite element methods when $\boldsymbol{u} \in H^{2}(\Omega)$. In this case $\boldsymbol{u}$ is continuous on $\bar{\Omega}$. By using the inverse inequality when $\Gamma_{h}$ is quasi-regular [5], it follows that there exists a constant $C$ such that

$$
\left\|\boldsymbol{u}-\boldsymbol{u}_{h}\right\|_{L^{\infty}(\Omega)} \leq C h^{1 / 2},
$$

and consequently $\left\|\boldsymbol{u}_{h}\right\|_{L^{\infty}(\Omega)}$ is bounded, independently of $h$. In order to simplify the presentation, we assume in the following that $\boldsymbol{u}_{h} . \boldsymbol{n}=0$ on the boundary $\partial \Omega$ of $\Omega$, but $\operatorname{div}\left(\boldsymbol{u}_{h}\right)$ is not necessarily vanishing. A consequence of $(2.1)$ and (3.4) is that

$$
\left\|\operatorname{div}\left(\boldsymbol{u}_{h}\right)\right\| \leq C h .
$$

In the following, we suppose that $\alpha$ is strictly positive. Then $(\mu, \omega)_{1}=_{\operatorname{def}} \epsilon \int_{\Omega} \nabla \mu \cdot \nabla \omega \mathrm{d} x+\alpha \int_{\partial \Omega} \mu \omega \mathrm{d} s$ is a scalar product on $H^{1}(\Omega)$ equivalent to the standard $H^{1}(\Omega)$ scalar product. In this case we can define the projector $R_{h}: \mu \in H^{1}(\Omega) \rightarrow R_{h} \mu \in V_{h}$ by:

$$
\left(\mu-R_{h} \mu, \omega\right)_{1}=0, \forall \omega \in V_{h}, \forall \mu \in H^{1}(\Omega),
$$


and it is well known that if the triangulation is regular, in the sense of [5], there exists a constant $C$ satisfying

$$
\left\|\mu-R_{h} \mu\right\|+h\left\|\nabla\left(\mu-R_{h} \mu\right)\right\| \leq C h^{2}\|\mu\|_{H^{2}(\Omega)} \quad \forall \mu \in H^{2}(\Omega)
$$

In order to prove convergence results, we introduce, as in [12] the following notations:

$$
\theta=\varphi_{h}-R_{h} \varphi \text { and } \rho=R_{h} \varphi-\varphi
$$

and we have $\theta+\rho=\varphi_{h}-\varphi$.

In order to establish some error estimates, we assume that the initial conditions $\varphi_{0}$ and $\varphi_{h}^{0}$ satisfy

$$
\varphi_{0} \in H^{2}(\Omega) \text { and } \varphi_{h}^{0}=R_{h} \varphi_{0} .
$$

Lemma 4.1. We assume that $\varphi \in C^{1}\left([0, T] ; H^{2}(\Omega)\right)$ and that there exists a constant $C$ independent of $h$ such that $\left\|\varphi_{h}\right\|_{L^{\infty}(\Omega)} \leq C, \forall t \in(0, T)\left(L^{\infty}\right.$-stability). Moreover we assume that Hypothesis (3.4) is satisfied, that $\boldsymbol{u}_{h} \cdot \boldsymbol{n}=0$ on $\partial \Omega$ and that the mesh $\Gamma_{h}$ is quasi-regular. Under theses assumptions, there exists a constant $\bar{C}$ independent of $h$ and $\epsilon$ which satisfies:

$$
\|\theta(t)\| \leq \mathrm{e}^{-\lambda_{1} t}\|\theta(0)\|+\int_{0}^{t}\left\|\rho_{t}(s)\right\| \mathrm{e}^{-\lambda_{1}(t-s)} \mathrm{d} s+\bar{C} h t, \quad 0<t<T,
$$

where $\rho_{t}=\frac{\mathrm{d}}{\mathrm{d} t} \rho$.

Proof. By taking $\psi=\theta$ in (2.5) and (3.6), we obtain:

$$
\int_{\Omega} \frac{\partial}{\partial t}\left(\varphi-\varphi_{h}\right) \theta \mathrm{d} x+\left(\varphi-\varphi_{h}, \theta\right)_{1}+\frac{1}{2} \int_{\Omega}\left((\theta-\bar{\theta})\left[\boldsymbol{u} \cdot \boldsymbol{\nabla} \varphi-\boldsymbol{u}_{h} \cdot \boldsymbol{\nabla} \varphi_{h}\right]+\left(\varphi_{h}-\bar{\varphi}_{h}\right) \boldsymbol{u}_{h} \cdot \boldsymbol{\nabla} \theta-(\varphi-\bar{\varphi}) \boldsymbol{u} \cdot \boldsymbol{\nabla} \theta\right) \mathrm{d} x=0
$$

In order to evaluate the first term above, we write:

$$
\begin{aligned}
S_{1} & =\int_{\Omega} \frac{\partial}{\partial t}\left(\varphi-\varphi_{h}\right) \theta \mathrm{d} x \\
& =\int_{\Omega} \frac{\partial}{\partial t}\left(\varphi-R_{h} \varphi\right) \theta \mathrm{d} x+\int_{\Omega} \frac{\partial}{\partial t}\left(R_{h} \varphi-\varphi_{h}\right) \theta \mathrm{d} x \\
& =-\int_{\Omega} \rho_{t} \theta \mathrm{d} x-\frac{1}{2} \frac{\mathrm{d}}{\mathrm{d} t}\|\theta\|^{2} .
\end{aligned}
$$

In order to evaluate the second term, we use (4.3) and we write:

$$
\begin{aligned}
S_{2} & =\left(\varphi-\varphi_{h}, \theta\right)_{1} \\
& =\left(\varphi-R_{h} \varphi, \theta\right)_{1}+\left(R_{h} \varphi-\varphi_{h}, \theta\right)_{1} \\
& =-(\theta, \theta)_{1} \leq-\lambda_{1}\|\theta\|^{2} .
\end{aligned}
$$


It remains to evaluate the third term. Integrating by parts and using (2.1) with $\boldsymbol{u}_{h} . \boldsymbol{n}=0$ on $\partial \Omega$, we obtain:

$$
\begin{aligned}
S_{3}= & \frac{1}{2} \int_{\Omega}\left((\theta-\bar{\theta})\left[\boldsymbol{u} \cdot \boldsymbol{\nabla} \varphi-\boldsymbol{u}_{h} \cdot \boldsymbol{\nabla} \varphi_{h}\right]+\left(\varphi_{h}-\bar{\varphi}_{h}\right) \boldsymbol{u}_{h} \cdot \boldsymbol{\nabla} \theta-(\varphi-\bar{\varphi}) \boldsymbol{u} \cdot \boldsymbol{\nabla} \theta\right) \mathrm{d} x \\
= & \frac{1}{2} \int_{\Omega}\left(2(\theta-\bar{\theta}) \boldsymbol{u} \cdot \boldsymbol{\nabla} \varphi-2(\theta-\bar{\theta}) \boldsymbol{u}_{h} \cdot \boldsymbol{\nabla} \varphi_{h}-\left(\varphi_{h}-\bar{\varphi}_{h}\right)(\theta-\bar{\theta}) \operatorname{div} \boldsymbol{u}_{h}\right) \mathrm{d} x \\
= & \frac{1}{2} \int_{\Omega}\left(2(\theta-\bar{\theta}) \boldsymbol{u} \cdot \boldsymbol{\nabla}\left(\varphi-R_{h} \varphi\right)+2(\theta-\bar{\theta}) \boldsymbol{u} \cdot \boldsymbol{\nabla}\left(R_{h} \varphi-\varphi_{h}\right)\right. \\
& \left.\quad+2(\theta-\bar{\theta})\left(\boldsymbol{u}-\boldsymbol{u}_{h}\right) \cdot \boldsymbol{\nabla} \varphi_{h}-(\theta-\bar{\theta})\left(\varphi_{h}-\bar{\varphi}_{h}\right) \operatorname{div} \boldsymbol{u}_{h}\right) \mathrm{d} x \\
= & \frac{1}{2} \int_{\Omega}\left(-2(\theta-\bar{\theta}) \boldsymbol{u} \cdot \boldsymbol{\nabla} \rho-\boldsymbol{u} \cdot \boldsymbol{\nabla} \theta^{2}+2(\theta-\bar{\theta})\left(\boldsymbol{u}-\boldsymbol{u}_{h}\right) \cdot \boldsymbol{\nabla} \varphi_{h}\right. \\
& \left.\quad-(\theta-\bar{\theta})\left(\varphi_{h}-\bar{\varphi}_{h}\right) \operatorname{div} \boldsymbol{u}_{h}\right) \mathrm{d} x \\
= & \frac{1}{2} \int_{\Omega}\left(-2(\theta-\bar{\theta}) \boldsymbol{u} \cdot \boldsymbol{\nabla} \rho+2(\theta-\bar{\theta})\left(\boldsymbol{u}-\boldsymbol{u}_{h}\right) \cdot \boldsymbol{\nabla} \varphi_{h}-(\theta-\bar{\theta})\left(\varphi_{h}-\bar{\varphi}_{h}\right) \operatorname{div} \boldsymbol{u}_{h}\right) \mathrm{d} x,
\end{aligned}
$$

and consequently:

$$
\left|S_{3}\right| \leq\left[\|\boldsymbol{u}\|_{L^{\infty}(\Omega)}\|\nabla \rho\|+\left\|\boldsymbol{u}-\boldsymbol{u}_{h}\right\|_{L^{2}(\Omega)}\left\|\nabla \varphi_{h}\right\|_{L^{\infty}(\Omega)}+\frac{1}{2}\left\|\varphi_{h}-\bar{\varphi}_{h}\right\|_{L^{\infty}(\Omega)}\left\|\operatorname{div} \boldsymbol{u}_{h}\right\|\right]\|\theta-\bar{\theta}\| .
$$

Taking into account the inverse inequality $\left\|\nabla \varphi_{h}\right\|_{L^{\infty}(\Omega)} \leq C h^{-1}\left\|\varphi_{h}\right\|_{L^{\infty}(\Omega)}$ (see [5]), the inequality $\|\bar{\theta}\| \leq\|\theta\|$ and the fact that $\left\|\varphi_{h}\right\|_{L^{\infty}(\Omega)}$ is assumed to be bounded, we obtain with (3.4)-(4.2):

$$
\left|S_{3}\right| \leq C(\|\nabla \rho\|+h)\|\theta\|
$$

where $C$ is a generic constant independent of $h$ and $t \in(0, T)$.

Since we assumed that $\varphi \in C^{1}\left([0, T] ; H^{2}(\Omega)\right)$, then by $(4.4),\|\nabla \rho\|$ is bounded with respect to $h$ and consequently:

$$
\left|S_{3}\right| \leq C h\|\theta\|
$$

Using Estimates $S_{1}, S_{2}, S_{3}$ we finally obtain:

$$
\frac{1}{2} \frac{\mathrm{d}}{\mathrm{d} t}\|\theta\|^{2}+\lambda_{1}\|\theta\|^{2} \leq\left(\left\|\rho_{t}\right\|+C h\right)\|\theta\|,
$$

which implies

$$
\frac{\mathrm{d}}{\mathrm{d} t}\|\theta\|+\lambda_{1}\|\theta\| \leq\left(\left\|\rho_{t}\right\|+C h\right)
$$

Setting $v(t)=\|\theta(t)\| \mathrm{e}^{\lambda_{1} t}$, we have $\frac{\mathrm{d}}{\mathrm{d} t} v(t)=\left(\frac{\mathrm{d}}{\mathrm{d} t}\|\theta\|+\lambda_{1}\|\theta\|\right) \mathrm{e}^{\lambda_{1} t} \leq\left(\left\|\rho_{t}\right\|+C h\right) \mathrm{e}^{\lambda_{1} t}$ and finally:

$$
\|\theta(t)\| \leq \mathrm{e}^{-\lambda_{1} t}\|\theta(0)\|+\int_{0}^{t}\left\|\rho_{t}(s)\right\| \mathrm{e}^{-\lambda_{1}(t-s)} \mathrm{d} s+\frac{C h}{\lambda_{1}}\left(1-\mathrm{e}^{-\lambda_{1} t}\right) .
$$

Theorem 4.1. We assume that $\varphi \in C^{1}\left([0, T] ; H^{2}(\Omega)\right)$ and that there exists a constant $C$ independent of $h$ such that $\left\|\varphi_{h}\right\|_{L^{\infty}(\Omega)} \leq C, \forall t \in(0, T)\left(L^{\infty}-\right.$ stability). Moreover we assume that Hypotheses (3.4), (4.6) are 
satisfied, that $\boldsymbol{u}_{h} \cdot \boldsymbol{n}=0$ on $\partial \Omega$ and that the mesh $\Gamma_{h}$ is quasi-regular. Under these assumptions there exists a constant $C_{1}$ independent of $h$ which satisfies:

$$
\left\|\varphi-\varphi_{h}\right\|_{L^{\infty}\left(0, T ; L^{2}(\Omega)\right)} \leq C_{1} h .
$$

Proof. From (4.4), (4.6) and Hypothesis $\varphi \in C^{1}\left([0, T] ; H^{2}(\Omega)\right)$, we have:

$$
\begin{aligned}
\|\rho(t)\| & \leq C h^{2} \text { and }\left(\int_{0}^{t}\left\|\rho_{t}(s)\right\|^{2} \mathrm{~d} s\right)^{\frac{1}{2}} \leq C h^{2} \text { for every } t \in(0, T), \\
\|\theta(0)\| & =\left\|\varphi_{h}(0)-\varphi(0)\right\| \leq C h^{2},
\end{aligned}
$$

where $C$ is a generic constant. Using Lemma 4.1 and the equality $\varphi_{h}-\varphi=\theta+\rho$, we easily prove inequality (4.8).

In order to estimate $\left\|\boldsymbol{\nabla}\left(\varphi-\varphi_{h}\right)\right\|_{L^{\infty}\left(0, T ; L^{2}(\Omega)\right)}$ we start by proving

Lemma 4.2. We assume the hypotheses of Lemma 4.1. Then there exists a constant $C$ which satisfies

$$
\left\|\theta_{t}\right\|^{2}+\frac{1}{2} \frac{\mathrm{d}}{\mathrm{d} t}\|\theta\|_{1}^{2} \leq\left\|\theta_{t}\right\|\left[\left\|\rho_{t}\right\|+C\left(h+\|\theta\|_{1}\right)\right] .
$$

where $\theta_{t}=\frac{\mathrm{d}}{\mathrm{d} t} \theta, \rho_{t}=\frac{\mathrm{d}}{\mathrm{d} t} \rho$.

Proof. By taking $\psi=\theta_{t}$ in (2.5) and (3.6) we obtain:

$$
\begin{aligned}
\int_{\Omega} \frac{\partial}{\partial t}(\varphi & \left.-\varphi_{h}\right) \theta_{t} \mathrm{~d} x+\left(\varphi-\varphi_{h}, \theta_{t}\right)_{1} \\
& +\frac{1}{2} \int_{\Omega}\left(\left(\theta_{t}-\bar{\theta}_{t}\right)\left[\boldsymbol{u} \cdot \boldsymbol{\nabla} \varphi-\boldsymbol{u}_{h} \cdot \boldsymbol{\nabla} \varphi_{h}\right]+\left(\varphi_{h}-\bar{\varphi}_{h}\right) \boldsymbol{u}_{h} \cdot \nabla \theta_{t}-(\varphi-\bar{\varphi}) \boldsymbol{u} \cdot \boldsymbol{\nabla} \theta_{t}\right) \mathrm{d} x=0 .
\end{aligned}
$$

In order to evaluate the first term above we write:

$$
\begin{aligned}
S_{1} & =\int_{\Omega} \frac{\partial}{\partial t}\left(\varphi-\varphi_{h}\right) \theta_{t} \mathrm{~d} x \\
& =\int_{\Omega} \frac{\partial}{\partial t}\left(\varphi-R_{h} \varphi\right) \theta_{t} \mathrm{~d} x+\int_{\Omega} \frac{\partial}{\partial t}\left(R_{h} \varphi-\varphi_{h}\right) \theta_{t} \mathrm{~d} x \\
& =-\int_{\Omega} \rho_{t} \theta_{t} \mathrm{~d} x-\left\|\theta_{t}\right\|^{2} .
\end{aligned}
$$

In order to evaluate the second term we write:

$$
\begin{aligned}
S_{2} & =\left(\varphi-\varphi_{h}, \theta_{t}\right)_{1} \\
& =\left(\varphi-R_{h} \varphi, \theta_{t}\right)_{1}-\frac{1}{2} \frac{\mathrm{d}}{\mathrm{d} t}\|\theta\|_{1}^{2} .
\end{aligned}
$$

The third term is evaluated like in Lemma 4.1:

$$
\begin{aligned}
S_{3}= & \frac{1}{2} \int_{\Omega}\left(\left(\theta_{t}-\bar{\theta}_{t}\right)\left[\boldsymbol{u} \cdot \boldsymbol{\nabla} \varphi-\boldsymbol{u}_{h} \cdot \boldsymbol{\nabla} \varphi_{h}\right]+\left(\varphi_{h}-\bar{\varphi}_{h}\right) \boldsymbol{u}_{h} \cdot \boldsymbol{\nabla} \theta_{t}-(\varphi-\bar{\varphi}) \boldsymbol{u} \cdot \boldsymbol{\nabla} \theta_{t}\right) \mathrm{d} x \\
= & \frac{1}{2} \int_{\Omega}\left(2\left(\theta_{t}-\bar{\theta}_{t}\right) \boldsymbol{u} \cdot \boldsymbol{\nabla} \varphi-2\left(\theta_{t}-\bar{\theta}_{t}\right) \boldsymbol{u}_{h} \cdot \boldsymbol{\nabla} \varphi_{h}-\left(\varphi_{h}-\bar{\varphi}_{h}\right)\left(\theta_{t}-\bar{\theta}_{t}\right) \operatorname{div} \boldsymbol{u}_{h}\right) \mathrm{d} x \\
= & \frac{1}{2} \int_{\Omega}\left(-2\left(\theta_{t}-\bar{\theta}_{t}\right) \boldsymbol{u} \cdot \boldsymbol{\nabla} \rho-2\left(\theta_{t}-\bar{\theta}_{t}\right) \boldsymbol{u} \cdot \boldsymbol{\nabla} \theta\right. \\
& \left.+2\left(\theta_{t}-\bar{\theta}_{t}\right)\left(\boldsymbol{u}-\boldsymbol{u}_{h}\right) \cdot \boldsymbol{\nabla} \varphi_{h}-\left(\varphi_{h}-\bar{\varphi}_{h}\right)\left(\theta_{t}-\bar{\theta}_{t}\right) \operatorname{div} \boldsymbol{u}_{h}\right) \mathrm{d} x .
\end{aligned}
$$


It follows with $\|\nabla \rho\| \leq C h$ (see (4.4)) and $\|\nabla \theta\|^{2} \leq \frac{1}{\epsilon}\|\theta\|_{1}^{2}$ that:

$$
\left|S_{3}\right| \leq\left[\|\boldsymbol{u}\|_{L^{\infty}(\Omega)} C\left(h+\|\theta\|_{1}\right)+\left\|\boldsymbol{u}-\boldsymbol{u}_{h}\right\|\left\|\nabla \varphi_{h}\right\|_{L^{\infty}(\Omega)}+\frac{1}{2}\left\|\operatorname{div} \boldsymbol{u}_{h}\right\|\left\|\left(\varphi_{h}-\bar{\varphi}_{h}\right)\right\|_{L^{\infty}(\Omega)}\right]\left\|\left(\theta_{t}-\bar{\theta}_{t}\right)\right\|
$$

and with (3.4), (4.1), and the inverse inequality $\left\|\nabla \varphi_{h}\right\|_{L^{\infty}(\Omega)} \leq C h^{-1}\left\|\varphi_{h}\right\|_{L^{\infty}(\Omega)}$, we obtain $\left|S_{3}\right| \leq C(h+$ $\left.\|\theta\|_{1}\right)\left\|\theta_{t}\right\|$ and finally the announced result of Lemma 4.2 .

Theorem 4.2. We assume that $\varphi \in C^{1}\left([0, T] ; H^{2}(\Omega)\right)$ and that there exists a constant $C$ independent of $h$ such that $\left\|\varphi_{h}\right\|_{L^{\infty}(\Omega)} \leq C, \forall t \in(0, T)$ ( $L^{\infty}-$ stability). Moreover we assume that Hypotheses (3.4), (4.6) are satisfied, that $\boldsymbol{u}_{h} \cdot \boldsymbol{n}=0$ on $\partial \Omega$ and that the mesh $\Gamma_{h}$ is quasi-regular. Under these assumptions, there exists a constant $C_{2}$ independent of $h$ which satisfies:

$$
\left\|\varphi-\varphi_{h}\right\|_{L^{\infty}\left(0, T ; H^{1}(\Omega)\right)} \leq C_{2} h .
$$

Proof. We have

$$
\begin{aligned}
\left\|\theta_{t}\right\|\left[\left\|\rho_{t}\right\|+C\left(h+\|\theta\|_{1}\right)\right] & \leq \frac{1}{2}\left\|\theta_{t}\right\|^{2}+\frac{1}{2}\left[\left\|\rho_{t}\right\|+C\left(h+\|\theta\|_{1}\right)\right]^{2} \\
& \leq \frac{1}{2}\left\|\theta_{t}\right\|^{2}+\left\|\rho_{t}\right\|^{2}+2 C^{2}\left(h^{2}+\|\theta\|_{1}^{2}\right) .
\end{aligned}
$$

The inequality of Lemma 4.2 implies, if $C$ is a generic constant, that

$$
\frac{1}{2}\left\|\theta_{t}\right\|^{2}+\frac{1}{2} \frac{\mathrm{d}}{\mathrm{d} t}\|\theta\|_{1}^{2} \leq C\left(h^{2}+\|\theta\|_{1}^{2}\right)+\left\|\rho_{t}\right\|^{2} .
$$

From this above relation we obtain $\|\theta(t)\|_{1}^{2} \leq C\left(\|\theta(0)\|_{1}^{2}+h^{2}+\int_{0}^{t}\left\|\rho_{t}(s)\right\|^{2} \mathrm{~d} s\right)$.

Since $\varphi \in C^{1}\left([0, T] ; H^{2}(\Omega)\right)$, there exists a constant $\mathrm{C}$ such that:

$$
\|\theta(t)\|_{1}^{2} \leq C\left(\|\theta(0)\|_{1}^{2}+h^{2}\right) \text { for every } t \in(0, T) .
$$

Relations (4.4) and (4.6) imply that $\|\theta(0)\|_{1} \leq C h$. Finally by (4.4): $\left\|\varphi-\varphi_{h}\right\|_{1}=\|\theta+\rho\|_{1} \leq C h$ for every $t \in[0, T]$.

\section{Discretization in time With A CONSERVAtive SCHEME}

As before, we assume that $\alpha$ is strictly positive. Let us consider a backward Euler scheme in order to discretize (3.6) in time. If $0=t_{0}<t_{1}<t_{2}<\ldots<t_{n}<\ldots<t_{N}=T$ is a discretization of the time interval $[0, T]$ and if we assume that we know the approximations $\varphi_{h}^{n} \simeq \varphi_{h}\left(t_{n}\right)$ at time $t_{n}$, we are looking for $\varphi_{h}^{n+1} \in V_{h}$ satisfying

$$
\begin{gathered}
\int_{\Omega} \frac{\varphi_{h}^{n+1}-\varphi_{h}^{n}}{t_{n+1}-t_{n}} \psi \mathrm{d} x+\epsilon \int_{\Omega} \nabla \varphi_{h}^{n+1} \cdot \nabla \psi \mathrm{d} x+\alpha \int_{\partial \Omega} \varphi_{h}^{n+1} \psi \mathrm{d} s \\
+\frac{1}{2} \int_{\Omega}\left(\boldsymbol{u}_{h} \cdot \nabla \varphi_{h}^{n+1}\right)(\psi-\bar{\psi}) \mathrm{d} x-\frac{1}{2} \int_{\Omega}\left(\boldsymbol{u}_{h} \cdot \nabla \psi\right)\left(\varphi_{h}^{n+1}-\bar{\varphi}_{h}^{n+1}\right) \mathrm{d} x \\
=\int_{\Omega} f\left(t^{n+1}\right) \psi \mathrm{d} x, \quad \forall \psi \in V_{h} .
\end{gathered}
$$


Remark 5.1. In practice, in order to solve Problem (5.1) with the finite element method we decompose $\varphi_{h}^{n+1}=$ $\bar{\varphi}_{h}^{n+1}+\widetilde{\varphi}_{h}^{n+1}$ and we introduce a Lagrange multiplier in order to take into account that $(\psi-\bar{\psi})$ has mean value zero as in (3.9)-(3.10)-(3.11).

Remark 5.2. When we take $\psi=\varphi_{h}^{n+1}$ in (5.1) we obtain:

$$
\left\|\varphi_{h}^{n+1}\right\|^{2}+\left(t_{n+1}-t_{n}\right)\left\|\varphi_{h}^{n+1}\right\|_{1}^{2} \leq \int_{\Omega} \varphi_{h}^{n+1} \varphi_{h}^{n} \mathrm{~d} x+\left(t_{n+1}-t_{n}\right)\left\|f\left(t^{n+1}\right)\right\|\left\|\varphi_{h}^{n+1}\right\|
$$

and it follows

$$
\left(1+\lambda_{1}\left(t_{n+1}-t_{n}\right)\right)\left\|\varphi_{h}^{n+1}\right\| \leq\left\|\varphi_{h}^{n}\right\|+\left(t_{n+1}-t_{n}\right)\left\|f\left(t^{n+1}\right)\right\| .
$$

Properties $(1 h),(2 h),(3 h)$ mentioned in Section 1 are satisfied with the scheme (5.1).

In order to establish an error estimate we proceed again like in [12]. We limit us to the case $\alpha>0$ and we set analogously to (4.5)

$$
\theta^{n}=\varphi_{h}^{n}-R_{h} \varphi\left(t_{n}\right) \text { and } \rho^{n}=R_{h} \varphi\left(t_{n}\right)-\varphi\left(t_{n}\right)
$$

In order to simplify the notations, we denote by

$$
\begin{aligned}
r_{n+1} & =t_{n+1}-t_{n} \text { and } \varphi^{n}=\varphi\left(t_{n}\right), \\
\bar{\partial} \theta^{n+1} & =\left(\theta^{n+1}-\theta^{n}\right) /\left(t_{n+1}-t_{n}\right) \\
\tau & =\max _{1 \leq n \leq N} r_{n} .
\end{aligned}
$$

Theorem 5.1. We assume that $\varphi \in C^{1}\left([0, T] ; H^{2}(\Omega)\right) \cap C^{2}\left([0, T] ; L^{2}(\Omega)\right)$ and that there exists a constant $C$ independent of $h$ and $n$ such that $\left\|\varphi_{h}^{n}\right\|_{L^{\infty}(\Omega)} \leq C,\left(L^{\infty}\right.$-stability). Moreover we assume that Hypotheses (3.4), (4.6) are satisfied, that $\boldsymbol{u}_{h} \cdot \boldsymbol{n}=0$ on $\partial \Omega$ and that the mesh $\Gamma_{h}$ is quasi-regular. Under these assumptions, there exists a constant $C_{3}$ independent of $h$ which satisfies:

$$
\left\|\varphi\left(t_{n}\right)-\varphi_{h}^{n}\right\|_{L^{2}(\Omega)} \leq C_{3}(h+\tau) \text { for every } 0<n \leq N .
$$

Proof. The proof of Theorem 5.1 is very similar to the proof of Theorem 4.1 via Lemma 4.1. By choosing $\psi=\theta^{n+1}$ in (2.5) and in (5.1), we obtain, with an integration by parts of the term $\frac{1}{2} \int_{\Omega}\left(\boldsymbol{u}_{h} \cdot \boldsymbol{\nabla} \psi\right) \varphi_{h}^{n+1} \mathrm{~d} x$ :

$$
\int_{\Omega} \bar{\partial} \theta^{n+1} \cdot \theta^{n+1} \mathrm{~d} x+\left\|\theta^{n+1}\right\|_{1}^{2}=\int_{\Omega}\left(\omega_{1}^{n+1}+\omega_{2}^{n+1}\right) \theta^{n+1} \mathrm{~d} x
$$

with

$$
\omega_{1}^{n+1}=\boldsymbol{u} \cdot \boldsymbol{\nabla} \varphi^{n+1}-\boldsymbol{u}_{h} \cdot \nabla \varphi_{h}^{n+1}-\frac{1}{2} \operatorname{div}\left(\boldsymbol{u}_{h}\right)\left(\varphi_{h}^{n+1}-\bar{\varphi}_{h}^{n+1}\right)
$$

and

$$
\omega_{2}^{n+1}=\frac{\partial \varphi}{\partial t}\left(t_{n+1}\right)-\bar{\partial} R_{h} \varphi^{n+1}
$$

The error estimate for $\int_{\Omega} \omega_{1}^{n+1} \theta^{n+1} \mathrm{~d} x$ is obtained as for $S_{3}$ in Lemma 4.1 by replacing $\theta$ by $\theta^{n+1}$, i.e.

$$
\left|\int_{\Omega} \omega_{1}^{n+1} \cdot \theta^{n+1} \mathrm{~d} x\right| \leq C\left(\left\|\nabla \rho^{n+1}\right\|+h\right)\left\|\theta^{n+1}\right\| \leq D h\left\|\theta^{n+1}\right\|,
$$


where $C, D$ are two constants independent of $h$ and $n$. The error estimate for $\int_{\Omega} \omega_{2}^{n+1} \theta^{n+1} \mathrm{~d} x$ follows from (4.4):

$$
\left|\int_{\Omega} \omega_{2}^{n+1} \theta^{n+1} \mathrm{~d} x\right| \leq\left|\int_{\Omega}\left(\frac{\partial \varphi}{\partial t}\left(t_{n+1}\right)-\bar{\partial} \varphi^{n+1}\right) \theta^{n+1} \mathrm{~d} x\right|+\left|\int_{\Omega} \bar{\partial} \rho^{n+1} \theta^{n+1} \mathrm{~d} x\right|
$$

and consequently, since we have assumed $\varphi \in C^{1}\left([0, T] ; H^{2}(\Omega)\right) \cap C^{2}\left([0, T] ; L^{2}(\Omega)\right)$ :

$$
\left|\int_{\Omega} \omega_{2}^{n+1} \theta^{n+1} \mathrm{~d} x\right| \leq C\left(r_{n+1}+r_{n+1} h^{2}\right)\left\|\theta^{n+1}\right\| \leq D \tau\left\|\theta^{n+1}\right\| .
$$

From (5.8), (5.9) and (5.10) we obtain:

$$
\left\|\theta^{n+1}\right\| \leq\left[\left\|\theta^{n}\right\|+C r_{n+1}(\tau+h)\right] .
$$

Taking into account that $\sum_{j=1}^{n} r_{j}=t_{n}$, we finally obtain:

$$
\left\|\theta^{n}\right\| \leq\left\|\theta^{0}\right\|+C t_{n}(\tau+h) \leq\left\|\theta^{0}\right\|+C T(\tau+h) .
$$

In order to complete the proof of Theorem 5.1, we use the same arguments as the ones in the proof of Theorem 4.1.

By choosing $\psi=\bar{\partial} \theta^{n+1}$ in (2.5) and in (5.1), like in Theorems 4.2 and 5.1, the following result is standard (see $[7,12])$ :

Theorem 5.2. We assume the hypotheses of Theorem 5.1. Then there exists a constant $C_{4}$ such that

$$
\left\|\nabla\left(\varphi\left(t_{n}\right)-\varphi_{h}^{n}\right)\right\|_{L^{2}(\Omega)} \leq C_{4}(h+\tau) \text { for every } 0<n \leq N .
$$

Remark 5.3. It is possible to improve the $L^{2}$ estimation (5.7) in Theorem 5.3 by $\left\|\varphi\left(t_{n}\right)-\varphi_{h}^{n}\right\| \leq C_{4}\left(h^{2}+\right.$ $\tau) \forall 0<n \leq N$, but under the stronger hypothesis $\left\|\nabla \varphi_{h}(t)\right\|_{L^{\infty}(\Omega)} \leq C \forall t \in(0, T)$ (the stability of the gradient). To prove this result, it is enough to replace the projection $R_{h}$ used in Lemma 4.1 by the projection $\tilde{R}_{h}: \mu \in H^{1}(\Omega) \rightarrow \tilde{R}_{h} \mu \in V_{h}$ defined by

$$
a\left(\mu-\tilde{R}_{h} \mu, \omega\right)=0, \forall \omega \in V_{h}, \forall \mu \in H^{1}(\Omega)
$$

where the coercive bilinear form $a(.,$.$) on H^{1}(\Omega)$ is given by:

$$
a(\mu, \omega)=\epsilon \int_{\Omega} \nabla \mu \cdot \nabla \omega \mathrm{d} x+\frac{1}{2} \int_{\Omega}(\boldsymbol{u} \cdot \nabla \mu)(\omega-\bar{\omega}) \mathrm{d} x-\frac{1}{2} \int_{\Omega}(\boldsymbol{u} \cdot \nabla \omega)(\mu-\bar{\mu}) \mathrm{d} x+\alpha \int_{\partial \Omega} \mu \omega \mathrm{d} s .
$$

\section{About Neumann boundary conditions}

If $\alpha=0$ then $(\mu, \omega)_{1}={ }_{\text {def }} \epsilon \int_{\Omega} \nabla \mu \cdot \nabla \omega \mathrm{d} x$ is a scalar product on $\widetilde{H}^{1}(\Omega)$. In this case we can define the operator $R_{h}: \mu \in \widetilde{H}^{1}(\Omega) \rightarrow R_{h} \mu \in \widetilde{V}_{h}$ by:

$$
\left(\mu-R_{h} \mu, \omega\right)_{1}=0, \forall \omega \in \widetilde{V}_{h}, \forall \mu \in \widetilde{H}^{1}(\Omega),
$$

and in this case $\lambda_{1}=\inf _{\mu \in \widetilde{H}^{1}(\Omega)} \frac{(\mu, \mu)_{1}}{\|\mu\|^{2}}$ is positive. Moreover we have seen that if we decompose $\varphi$ and $\varphi_{h}$ by $\varphi=\bar{\varphi}+\widetilde{\varphi}$ and $\varphi_{h}=\bar{\varphi}_{h}+\widetilde{\varphi}_{h}$, then the equations for $\widetilde{\varphi}$ and $\bar{\varphi}$ are not coupled and analogously for $\widetilde{\varphi}_{h}$ and $\bar{\varphi}_{h}$. By defining $\theta=\widetilde{\varphi}_{h}-R_{h} \widetilde{\varphi}$ and $\rho=R_{h} \widetilde{\varphi}-\widetilde{\varphi}$, then Lemmas 4.1 and 4.2 remain true for functions with zero meanvalue and allow to obtain Theorems 4.1,4.2, 5.1 and 5.2 even if $\alpha=0$. 


\section{Numerical RESUlts}

We now check numerically that the conservative scheme presented in this article has the desired properties, even if the stabilization terms (3.3) are added into (3.6). Let $\Omega \subset \mathbb{R}^{3}$ be the domain $[-1,1]^{2} \times[-0.1,0.1]$ and

$$
\boldsymbol{u}(x, y, z)=\left(-\cos \left(\frac{3 \pi x}{2}\right) \sin \left(\frac{3 \pi y}{2}\right), \sin \left(\frac{3 \pi x}{2}\right) \cos \left(\frac{3 \pi y}{2}\right), 0\right) .
$$

It is easy to remark that $\boldsymbol{u} \cdot \boldsymbol{n}=0$ on $\partial \Omega$ and that $\operatorname{div} \boldsymbol{u}=0$. We also define the following exchange coefficient

$$
\alpha=\left\{\begin{array}{l}
1 \text { if }|z|<0.1 \\
0 \text { if }|z|=0.1
\end{array}\right.
$$

which implies that the domain is isolated on its top and bottom and in this particular case, the flow is twodimensional. We numerically solve the following problem: find $\varphi:(0, T) \times \Omega \rightarrow \mathbb{R}$ such that

$$
\left\{\begin{aligned}
\frac{\partial \varphi}{\partial t}-\epsilon \Delta \varphi+\boldsymbol{u} \cdot \nabla \varphi & =f & & \text { in } \Omega \\
\epsilon \frac{\partial \varphi}{\partial \boldsymbol{n}} & =-\alpha \varphi & & \text { on } \partial \Omega \\
\varphi(0) & =\varphi_{0} . & &
\end{aligned}\right.
$$

Let $\mathcal{T}_{h}$ be a uniform discretization of the domain $\Omega$ such that $h:=\max _{K \in \mathcal{T}_{h}}(\operatorname{diam}(K))=0.2, \Delta t=T / N$, $t_{n}=n \Delta t, n=0, \ldots, N$ and $V_{h}$ the space of piecewise linear finite elements defined on $\mathcal{T}_{h}$. The space-time discretization using the backward Euler method in time of (6.3) becomes: given $\varphi_{h}^{0}=\varphi_{0}$, for $n=0, \ldots, N-1$, we are looking for $\varphi_{h}^{n+1} \in V_{h}$ satisfying

$$
\left\{\begin{array}{c}
\int_{\Omega} \frac{\varphi_{h}^{n+1}-\varphi_{h}^{n}}{\Delta t} \psi_{h} \mathrm{~d} x+\int_{\Omega} \epsilon \boldsymbol{\nabla} \varphi_{h}^{n+1} \cdot \nabla \psi_{h} \mathrm{~d} x+\int_{\Omega} L\left(\boldsymbol{u}_{h}, \varphi_{h}^{n+1}, \psi_{h}\right) \mathrm{d} x \\
+\int_{\partial \Omega} \alpha \varphi_{h}^{n+1} \psi_{h} \mathrm{~d} s+\sum_{K \in \mathcal{T}_{h}} \int_{K} \beta_{1} \delta_{K} \frac{h_{K}}{\left\|\boldsymbol{u}_{h}\right\|}\left(\boldsymbol{u}_{h} \cdot \boldsymbol{\nabla} \varphi_{h}^{n+1}\right)\left(\boldsymbol{u}_{h} \cdot \boldsymbol{\nabla} \psi_{h}\right) \mathrm{d} x \\
\quad+\sum_{K \in \mathcal{T}_{h}} \int_{K} \beta_{2} \delta_{K} h_{K}\left\|\boldsymbol{u}_{h}\right\|\left(\boldsymbol{\nabla} \varphi_{h}^{n+1} \cdot \nabla \psi_{h}\right) \mathrm{d} x=\int_{\Omega} f^{n+1} \psi_{h} \mathrm{~d} x
\end{array}\right.
$$

for all $\psi_{h} \in V_{h}$, where $\beta_{1}$ is a stabilization parameter, $\beta_{2}$ an artificial diffusion parameter, $\delta_{K}$ is a function of local Péclet number $\mathbb{P} e_{K}$, i.e. $\delta_{K}=1$ if $\mathbb{P} e_{K} \geq 1$ and $\delta_{K}=\mathbb{P} e_{K}$ if not. $\operatorname{In}(6.4), L\left(\boldsymbol{u}_{h}, \varphi_{h}^{n+1}, \psi_{h}\right)$ is a discretization of the convective term, where $\boldsymbol{u}_{h}$ is an approximation of the velocity field (6.1). In our computation, $\boldsymbol{u}_{h}$ is obtained using a $\mathbb{P}_{1}-\mathbb{P}_{1}$ stabilized stationary Navier-Stokes solver in which the force term is such that (6.1) is a solution of the Navier-Stokes equations with pressure $p(x, y, z)=\frac{1}{4}(\cos (3 \pi x)+\cos (3 \pi y))$. The velocity field $\boldsymbol{u}_{h}$ is computed only once, before solving (6.3), and then used at each time step for the computation of $\varphi_{h}^{n+1}$.

In (6.4), we have added a SUPG stabilization term and an artificial diffusion term, because $h_{K}>\epsilon /\|\boldsymbol{u}\|_{L^{2}(K)}$. These stabilization terms do not influence the conservation of the integral, because both terms vanish when the test function $\psi_{h} \equiv 1$ is taken. Nevertheless we have to take them into account for $L^{2}$ stability verification, because they do not vanish when $\psi_{h} \equiv \varphi_{h}^{n+1}$. Actually, both are positive and contribute to stabilize the scheme.

We describe here the conservation properties that we claim our scheme satisfies numerically. The first one is the conservation of the integral, which states that

$$
\frac{\mathrm{d}}{\mathrm{d} t} \int_{\Omega} \varphi \mathrm{d} x=\int_{\Omega} f \mathrm{~d} x+\int_{\partial \Omega} \alpha\left(\varphi_{r}-\varphi\right) \mathrm{d} s .
$$


In our numerical tests, $\varphi_{r} \equiv 0$ and using backward Euler for time discretization of this relation, we obtain the discrete integral conservation: for $n=0, \ldots, N-1$

$$
\int_{\Omega} \varphi_{h}^{n+1} \mathrm{~d} x+\Delta t \int_{\partial \Omega} \alpha \varphi_{h}^{n+1} \mathrm{~d} s=\int_{\Omega} \varphi_{h}^{n}+\Delta t \int_{\Omega} f^{n+1} \mathrm{~d} x .
$$

The $L^{2}$-stability has not to take into account the convective term as shown in (2.7), (2.8) for the exact equation in which $\int_{\Omega}(\boldsymbol{u} \cdot \nabla \varphi) \varphi \mathrm{d} x=0$. In order to quantify the effect of the approximation $\int_{\Omega} L_{h}\left(\boldsymbol{u}_{h}, \varphi_{h}, \varphi_{h}\right) \mathrm{d} x$ in scheme (6.4), we propose to evaluate the $L^{2}$ stability of the approximate problem with the following criterion. We take $\psi_{h}=\varphi_{h}^{n+1}$ in (6.4) and we remove the convection term to obtain

$$
\begin{gathered}
\left\|\varphi_{h}^{n+1}\right\|_{L^{2}(\Omega)}^{2}+\Delta t\left(\int_{\partial \Omega} \alpha\left(\varphi_{h}^{n+1}\right)^{2} \mathrm{~d} s+\int_{\Omega} \epsilon\left|\nabla \varphi_{h}^{n+1}\right|^{2} \mathrm{~d} x\right)+\Delta t\left(S_{1}\left(\varphi_{h}^{n+1}, \varphi_{h}^{n+1}\right)+S_{2}\left(\varphi_{h}^{n+1}, \varphi_{h}^{n+1}\right)\right) \\
=\int_{\Omega} \varphi_{h}^{n+1} \varphi_{h}^{n} \mathrm{~d} x+\Delta t \int_{\Omega} f^{n+1} \varphi_{h}^{n+1} \mathrm{~d} x
\end{gathered}
$$

where

$$
S_{1}\left(\varphi_{h}, \psi_{h}\right)=\sum_{K \in \mathcal{T}_{h}} \int_{K} \beta_{1} \delta_{K} \frac{h_{K}}{\left\|\boldsymbol{u}_{h}\right\|}\left(\boldsymbol{u}_{h} \cdot \nabla \varphi_{h}\right)\left(\boldsymbol{u}_{h} \cdot \nabla \psi_{h}\right) \mathrm{d} x
$$

and

$$
S_{2}\left(\varphi_{h}, \psi_{h}\right)=\sum_{K \in \mathcal{T}_{h}} \int_{K} \beta_{2} \delta_{K} h_{K}\left\|\boldsymbol{u}_{h}\right\|\left(\nabla \varphi_{h} \cdot \nabla \psi_{h}\right) \mathrm{d} x .
$$

Remark that $S_{1}\left(\varphi_{h}, \varphi_{h}\right)$ and $S_{2}\left(\varphi_{h}, \varphi_{h}\right)$ are positive and contribute to the $L^{2}$-stability. If follows that Equality (6.6), together with the Cauchy-Schwarz inequality, implies that

$$
\left\|\varphi_{h}^{n+1}\right\|^{2}+\Delta t\left\|\varphi_{h}^{n+1}\right\|_{1}^{2} \leq\left\|\varphi_{h}^{n+1}\right\| \cdot\left\|\varphi_{h}^{n}\right\|+\Delta t\left\|f^{n+1}\right\| \cdot\left\|\varphi_{h}^{n+1}\right\| .
$$

By using the fact that $\lambda_{1 h}=\inf _{v_{h} \in V_{h}} \frac{\left\|v_{h}\right\|_{1}^{2}}{\left\|v_{h}\right\|^{2}} \geq \lambda_{1}$, we obtain

$$
\left(1+\lambda_{1} \Delta t\right)\left\|\varphi_{h}^{n+1}\right\| \leq\left\|\varphi_{h}^{n}\right\|+\Delta t\left\|f^{n+1}\right\|
$$

or

$$
\frac{\left\|\varphi_{h}^{n+1}\right\|-\left\|\varphi_{h}^{n}\right\|}{\Delta t}+\lambda_{1}\left\|\varphi_{h}^{n+1}\right\| \leq\left\|f^{n+1}\right\|
$$

which is the backward discretization in time of (2.12) and proves that our scheme is $L^{2}$ stable.

Finally, the third property is the conservation of a constant solution, i.e.

$$
\text { when } f=0 \text {, then } \varphi_{h}=\text { constant is a stationary solution of (6.4). }
$$

We now focus on the discretization of the convective term $L\left(\boldsymbol{u}_{h}, \varphi_{h}, \psi_{h}\right)$. We recall that they are mainly four standard possiblities other than the scheme proposed in this article

L1. $L\left(\boldsymbol{u}_{h}, \varphi_{h}, \psi_{h}\right)=\left(\boldsymbol{u}_{h} \cdot \nabla \varphi_{h}\right) \psi_{h}$,

L2. $L\left(\boldsymbol{u}_{h}, \varphi_{h}, \psi_{h}\right)=-\left(\boldsymbol{u}_{h} \cdot \nabla \psi_{h}\right) \varphi_{h}$,

L3. $L\left(\boldsymbol{u}_{h}, \varphi_{h}, \psi_{h}\right)=\operatorname{div}\left(\boldsymbol{u}_{h} \varphi_{h}\right) \psi_{h}$,

L4. $L\left(\boldsymbol{u}_{h}, \varphi_{h}, \psi_{h}\right)=\frac{1}{2}\left(\boldsymbol{u}_{h} \cdot \nabla \varphi_{h}\right) \psi_{h}-\frac{1}{2}\left(\boldsymbol{u}_{h} \cdot \nabla \psi_{h}\right) \varphi_{h}$,

and our scheme is

L5. $L\left(\boldsymbol{u}_{h}, \varphi_{h}, \psi_{h}\right)=\frac{1}{2}\left(\boldsymbol{u}_{h} \cdot \nabla \varphi_{h}\right)\left(\psi_{h}-\bar{\psi}_{h}\right)-\frac{1}{2}\left(\boldsymbol{u}_{h} \cdot \nabla \psi_{h}\right)\left(\varphi_{h}-\bar{\varphi}_{h}\right)$. 
Due to the fact that $\operatorname{div} \boldsymbol{u}_{h}$ is not equal to zero, each of the first four discretization conserves in principle and a priori only one of the three desired properties, i.e. (6.5), (6.6), (6.8). Of course, scheme L5 is the only one which conserves the three properties. We recall that Table 1 summarizes the conservated properties of each $L\left(\boldsymbol{u}_{h}, \varphi_{h}, \psi_{h}\right)$.

To check the (6.5) and (6.6) equalities, we compute $f$ and $\varphi_{0}$ in (6.3) so that the solution $\varphi$ is given by

$$
\varphi(t, x, y, z)=\left(1-\mathrm{e}^{-\lambda t}\right)\left[\frac{\cos (x)-\cos (1)}{\epsilon}+\sin (1)\right]\left[\frac{\cos (y)-\cos (1)}{\epsilon}+\sin (1)\right]
$$

with $\lambda=0.005$. With this right hand side $f$, we compute $\varphi_{h}^{n+1}$ solution of (6.4) with $\alpha$ defined in (6.2) and $\epsilon=10^{-5}$. For numerical approximation, we use $\Delta t=1$ and make 3000 iterations. At each time step $n$, we compute the quantity

$$
\Delta P 1(n)=\frac{\left|I_{1}-I_{2}\right|}{\left|I_{1}\right|}
$$

where $I_{1}, I_{2}$ are respectively the left-hand side and right-hand side of (6.5). We note that if the integral conservation property is satisfied, $\Delta P 1(n)=0$ for $n=0, \ldots, N-1$. Similary, for $L^{2}$ stability, we compute at each time step the estimator

$$
\Delta P 2(n)=\frac{\left|J_{1}-J_{2}\right|}{\left|J_{1}\right|}
$$

where $J_{1}, J_{2}$ are respectively the left-hand side and right-hand side of (6.6). If $\Delta P 2(n)=0$, then the discrete $L^{2}$ stability is achieved.

Let $\varphi_{r} \equiv 10, \epsilon, \alpha$ and $\boldsymbol{u}$ as before. To verify the conservation of constant solution, we solve the following problem

$$
\left\{\begin{aligned}
\frac{\partial \varphi}{\partial t}-\epsilon \Delta \varphi+\boldsymbol{u} \cdot \boldsymbol{\nabla} \varphi & =0 & & \text { in } \Omega \\
\epsilon \frac{\partial \varphi}{\partial \boldsymbol{n}} & =\alpha\left(\varphi_{r}-\varphi\right) & & \text { on } \partial \Omega \\
\varphi(0) & =\varphi_{r}, & &
\end{aligned}\right.
$$

and the solution is $\varphi \equiv \varphi_{r}$ for every $t$. Of course, we adapt numerical scheme (6.4) to problem (6.11) by adding $\int_{\partial \Omega} \varphi_{r} \psi_{h} \mathrm{~d} x$ to the right hand side of (6.4). The estimator we use to check the conservation of constant solution at each time step $n=0, \ldots, N-1$ is

$$
\Delta P 3(n)=\frac{\left\|\varphi_{r}-\varphi_{h}^{n+1}\right\|_{L^{\infty}}}{10} .
$$

Defining

- $\Pi_{1}=\max _{0 \leq n \leq N-1} \Delta P 1(n)$,

- $\Pi_{2}=\max _{0 \leq n \leq N-1} \Delta P 2(n)$,

- $\Pi_{3}=\max _{0 \leq n \leq N-1} \Delta P 3(n)$,

results are shown in Table 2 .

The results of Table 2 exactly match the claims in Table 1. We can also notice that the numerical scheme L5 is the only one which numerically satisfies the three conservation properties up to computer precision.

We now focus on error estimates for our new scheme. The exact solution we use to numerically verify Theorems 5.1 and 5.2 is the function defined in (6.9) with $\epsilon=\lambda=0.1$. With this value of $\epsilon$, we do not have to stabilize the numerical scheme. We have performed our computations on a structured mesh of parameter $h=0.1,0.05,0.025$ and 0.0125 . We set $T=4 \times 10^{-2}[\mathrm{~s}], \Delta t=h^{2}$ and compute $\left\|\varphi(T)-\varphi_{h}^{n}(T)\right\|_{L^{2}(\Omega)}$ and $\left\|\boldsymbol{\nabla}\left(\varphi(T)-\varphi_{h}^{n}(T)\right)\right\|_{L^{2}(\Omega)}$ for each $h$. The approximated velocity field $\boldsymbol{u}_{h}$ is computed as for the verification of properties P1 to P3. We obtained the results of Figure 1, which show that $\left\|\varphi(T)-\varphi_{h}^{n}(T)\right\|_{L^{2}(\Omega)}$ is of order two and that $\left\|\boldsymbol{\nabla}\left(\varphi(T)-\varphi_{h}^{n}(T)\right)\right\|_{L^{2}(\Omega)}$ is of order one. This shows that error estimates of Theorem 5.2 are optimal, but that we need stronger hypotheses in Theorem 5.1 to ensure the convergence of order two for the $L^{2}$ norm of the solution (see Rem. 5.3). 
TABLE 2. Numerical verification of the properties 1 to 3 .

\begin{tabular}{|l|c|c|c|}
\hline$L\left(\boldsymbol{u}_{h}, \varphi_{h}, \psi_{h}\right)$ & $\Pi_{1}$ & $\Pi_{2}$ & $\Pi_{3}$ \\
\hline L1 & $1.56 \times 10^{-4}$ & 0.0015 & $1.50 \times 10^{-10}$ \\
L2 & $4.17 \times 10^{-11}$ & 0.0014 & 0.0035 \\
L3 & $4.02 \times 10^{-11}$ & 0.0015 & 0.0035 \\
L4 & $8.48 \times 10^{-5}$ & $1.21 \times 10^{-12}$ & 0.0018 \\
L5 & $1.14 \times 10^{-11}$ & $3.38 \times 10^{-12}$ & $7.11 \times 10^{-14}$ \\
\hline
\end{tabular}

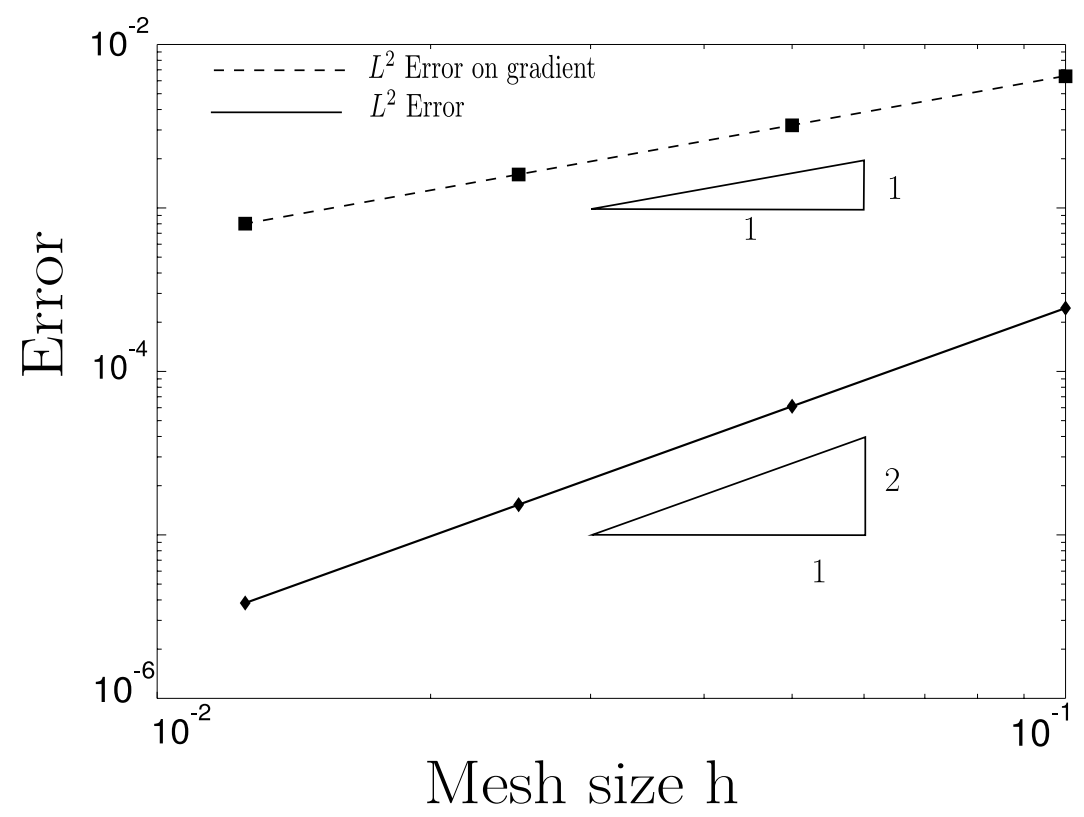

FiguRE 1. $\left\|\varphi(T)-\varphi_{h}^{n}(T)\right\|_{L^{2}(\Omega)}$ and $\left\|\nabla\left(\varphi(T)-\varphi_{h}^{n}(T)\right)\right\|_{L^{2}(\Omega)}$ for various mesh size $h$.

\section{Conclusion}

In this work, we have developed a new finite element numerical scheme for a convection-diffusion equation, which numerically conserves the integral, is $L^{2}$ stable, and conserves the constant solution even if the given convection field $\boldsymbol{u}_{h}$ is not completely divergence-free. We compare this new scheme with standard discretizations of the convective term and we show that only our scheme is able to conserve the three properties simultaneously. We have also derived a priori error estimates for this scheme and the numerical results show that these bounds are optimal. We haven't observe any numerical drawbacks for this scheme, except that the linear system associated with it has two full rows and two full colums in addition to those in the classical schemes. However, it has almost no incidence on CPU time. Thus we claim that only the finite element numerical scheme corresponding to L5 is efficient for numerical applications coupling the incompressible Navier-Stokes equations with the convectiondiffusion equation, as in $[8,9]$.

\section{REFERENCES}

[1] P. Angot, V. Dolej, M. Feistauer and J. Felcman, Analysis of a combined barycentric finite volumenonconforming finite element method for nonlinear convection-diffusion problems, Applications of Mathematics, vol. 43. Kluwer Academic Publishers-Plenum Publishers (1998) 263-310.

[2] I. Babuska and J. Osborn, Eigenvalue problems, Handbook of Numerical Analysis, vol. 2. Elsevier (1991) 641-787. 
[3] A. Brooks and T. Hughes, Streamline upwind/Petrov-Galerkin formulations for convection dominated flows with particular emphasis on the incompressible Navier-Stokes equations. Comput. Methods Appl. Mech. Engrg. 32 (1982) 199-259

[4] E. Burman and P.Hansbo, Edge stabilization for Galerkin approximations of convection-diffusion-reaction problems. Comput. Methods Appl. Mech. Engrg. 193 (2004) 1437-1453

[5] P.G. Ciarlet, The finite element method for elliptic problems. North-Holland Publishing Company (1978).

[6] R. Dautray and J.-L. Lions, Chap XVIII. Evolution Problems: Variational Methods, Math. Anal. and Numer. Methods Sci. Technology. vol. 5, Springer-Verlag, Heidelberg (2000) 467-680.

[7] A. Ern and J.-L. Guermond, Elements finis: Théorie, applications, mise en oeuvre. Springer-Verlag (2002).

[8] S. Flotron, Simulations numériques de phénomènes MHD-thermique avec interface libre dans l'électrolyse de l'aluminium, Ph.D. Thesis, EPFL, Switzerland, expected in (2013).

[9] T. Hofer, Numerical Simulation and optimization of the alumina distribution in an aluminium electrolysis pot, Ph.D. Thesis, Thesis No. 5023, EPFL, Switzerland (2011).

[10] A. Quarteroni and A. Valli, Numerical approximation of partial differential equations. Springer Series in Computational Mathematics (1997).

[11] R. Temam, Navier-Stokes equations. North-Holland (1984).

[12] V. Thomée, Galerkin Finite Element Methods for Parabolic Problems. Springer Series in Computational Mathematics. SpringerVerlag Berlin Heidelberg, New York (1997). 